\title{
Detection and characterization of single nanoparticles by interferometric phase modulated ellipsometry
}

\author{
F. Barroso a , S. Bosch a, N. Tort a , O. Arteaga a , J. Sancho-Parramon ${ }^{\text {b }}$, E. Jover a , E. Bertran ${ }^{\text {a }}$, A. Canillas ${ }^{\text {a,* }}$ \\ a Universitat de Barcelona, IN²UB, Dep. Física Aplicada i Òptica, c/Martí i Franqués 1, 08028, Spain \\ ${ }^{\mathrm{b}}$ Rudjer Boskovic Institute, Bijenicka c. 54, Zagreb 10002, Croatia
}

\section{A R T I C L E I N F O}

Available online $\mathrm{xxxx}$

Keywords:

Polarimetry

Interferometry

Phase-modulated ellipsometry

Nanoparticles detection

\begin{abstract}
A B S T R A C T
We introduce a new measurement system called Nanopolar interferometer devoted to monitor and characterize single nanoparticles which is based on the interferometric phase modulated ellipsometry technique. The system collects the backscattered light by the particles in the solid angle subtended by a microscope objective and then analyses its frequency components. The results for the detection of $2 \mu \mathrm{m}$ and $50 \mathrm{~nm}$ particles are explained in terms of a cross polarization effect of the polarization vectors when the beam converts from divergent to parallel in the microscope objective. This explanation is supported with the results of the optical modelling using the exact Mie theory for the light scattered by the particles.
\end{abstract}

(c) 2010 Elsevier B.V. All rights reserved.

\section{Introduction}

The increasing use of nanoscale products and materials in technological applications demands precise sampling techniques for the reliable detection, characterization, classification and tracking of nanoscopic matter. Common optical methods for particle detection rely on the measurement of the scattered light from the particles (optical particle counters, nephelometers, etc.). The sensitivity of these systems decreases remarkably when decreasing the number of particles, because the scattering intensity from a particle scales with the sixth power of the particle size, making impossible the detection of single nanoparticles.

Recently, two optical schemes based on interferometric techniques have been shown to be able to detect single nanoparticles below $10 \mathrm{~nm}$. Thus, the combination of interferometry with confocal microscopy using a supercontinuum white-light laser source allows identifying single metal nanoparticles through its plasmon resonance spectrum [1]. Also, the combination of interferometry with a split photodetector that generates a background free signal, allows to resolve individual particles and even to discriminate from other particles in real time [2]. The two methods are based on the detection of changes in the amplitude of the electric field governed by the interferometric term that scales as the 3rd power of the particle size. The additional use of polarized light in these interferometric setups appears as a promising way to increase the amount of information obtained, in such a way that, not only the size of the particle but also the shape and its dielectric properties could be accessible.

\footnotetext{
* Corresponding author. Tel.: +34 934037089; fax: +34 934039219. E-mail address: acanillas@ub.edu (A. Canillas).
}

This idea has motivated the work presented in this paper. Here we introduce a new measurement system, called Nanopolar interferometer, devoted to monitor and characterize single nanoparticles. It is based on the detection of the polarized backscattered light from the particles in an experimental setup that combines interferometry and phase-modulated ellipsometry. The results for the detection of $2 \mu \mathrm{m}$ and $50 \mathrm{~nm}$ size particles are presented and discussed with the help of an optical modelling of the light scattered by the particles.

\section{Principle}

The Nanopolar interferometer combines a modified TwymanGreen interferometer with a phase-modulated ellipsometer to detect small particles. The Jones vector, $J_{o}$, at the detector can be derived from the expression:

$J_{o}(t)=T_{A} T_{I} T_{P S G}(t) J_{i}$

where $J_{i}$ represents an incident Stokes vector, $T_{P S G}(t)$ the Jones matrix of the polarization sate generator (PSG), formed by a polarizer and a photoelastic modulator which gives a time-dependent phase retardation to the light beam, $T_{I}$ the Mueller matrix of the interferometer including the sample and $T_{A}$ the Mueller matrix of the analyzer. The expressions for $T_{P S G}(t)$ and $T_{A}$ are well known [3] whereas the expression of $T_{I}$ depends on the specific arrangement of the sample in the interferometer path. In our design we have substituted one of the mirrors of the interferometer by an oil-immersion microscope objective. This objective focuses the light beam into a fused silica microfluidic channel containing the particles, which are immersed in a liquid of refractive index $n_{p}$. The measurement system allows two modes of operation which correspond to the detection of the intensity 
of one beam or two beams of the interferometer; we call these modes single mode and interferometric mode respectively.

In the single mode the system detects the light backscattered by the particles and the objective (mainly by the oil/fused silica interface), thus, $T_{I}$ can be expressed as:

$T_{I}=T_{o b}+e^{i \delta_{p}} T_{o t} T_{p}$

where $T_{o b}$ represents the Jones matrix of the objective, $T_{p}$ Jones matrix of the particles for the solid angle subtended by the objective, $T_{o t}$ the transmission Jones matrix of the objective, which takes into account the bending of the polarization vectors when a divergent beam is converted into a parallel one [4], and $\delta_{p}$ the phase shift between the light backscattered by the objective and the particles. $T_{p}$ is function of the numerical aperture (N.A.) of the objective and may be obtained from the amplitude scattering matrix $S_{p}$ of the particles:

$T_{p}=\int_{0}^{2 \pi} \int_{\pi}^{\pi-\alpha} S_{p}(\theta, \phi) d \theta d \phi$,

$\phi$ being the azimuthal angle and $\theta$ the scattering angle [5(a)]. $\alpha$ can be obtained to a first approximation from the relation $n_{p} \sin \alpha=$ N.A. but it depends on the matching of the refractive index of the immersion oil, the microfluidic channel and the carrying fluid.

In the interferometric mode we allow the second beam to recombine with the first one and reach the detector, then:

$T_{I}=T_{o b}+e^{i \delta_{p}} T_{o t} T_{p}+e^{i \delta_{2}} T_{2}$,

where $\delta_{2}$ takes into account a phase shift between the two beams and $T_{2}$ includes all the effects of the optical elements placed in the second beam. In this operation mode a background-free detection would be possible if the destructive interference condition $T_{o b}+e^{i \delta_{2}} T_{2}=0$ is reached. However, the ideal condition which enhances the sensitivity to the passage of the nanoparticles will be attained for a constructive interference of the electric fields scattered by the nanoparticles and by the objective.

A phase modulated ellipsometer measures the ratios $R_{\omega}$ and $R_{2 \omega}$ of the detected intensity defined as the ratios of the fundamental and second harmonic components to the dc component, respectively [6]. Because the measurement corresponds to intensities these ratios are easily expressed in terms of the Mueller matrix, $M_{l}$, of the interferometric arrangement. In particular, for the orientations considered in the present work $\left(P=-45^{\circ}, \mathrm{M}=0^{\circ}\right.$, and $\left.\mathrm{A}=-45^{\circ}\right)$ and working with the $\mathrm{J}_{0}(A)=0$ condition, these ratios are $[5(\mathrm{~b})]$ :

$$
\begin{aligned}
& R_{\omega}=2 J_{1}(\mathrm{~A}) \frac{-m_{I(3,4)}+m_{I(1,4)}}{m_{I(1,1)}-m_{I(3,1)}}, \\
& R_{2 \omega}=2 J_{2}(\mathrm{~A}) \frac{m_{I(3,3)}-m_{I(1,3)}}{m_{I(1,1)}-m_{I(3,1)}}
\end{aligned}
$$

$J_{n}(A)$ being the nth Bessel function of the amplitude modulation, $A$, and $\mathrm{m}_{\mathrm{I}(\mathrm{i}, \mathrm{j})}$ the Mueller matrix elements of $\mathrm{M}_{\mathrm{I}}$. The correspondence between the Jones matrix $T_{I}$ and the Mueller matrix $M_{I}$ is straightforward if $M_{I}$ is a Mueller-Jones matrix [7]. Also $T_{p}$ has its corresponding Mueller Jones matrix, $\mathrm{M}_{\mathrm{p}}$, defined in terms of the scattering matrix [5(a)].

\section{Experimental}

\subsection{Optical set-up}

Fig. 1 shows a schema of the Nanopolar interferometer constructed in our laboratory. The light source is a $532 \mathrm{~nm}$ laser with variable power (150 mW, Cobolt Samba). The laser beam is collimated with a beam expanding lens (microscope objective), a pinhole and a collimating lens and then directed to the PSG. The collimated beam is then split by a non polarizing beam splitter into two perpendicular paths. One path will serve for optional interferometric recombination and the other path is focused with an oil-immersion objective $(\mathrm{NA}=1.35)$ onto a microfluidic channel carrying the particles. The backscattered light from single particles is collected with the same objective and sent back through the beam splitter to the analyzer. Finally, the image of the microfluidic channel is formed with the help of a tube lens on the photomultiplier tube (PMT) detector equipped with an interferential filter. Also a CCD camera is used in the image plane to monitor the passage of big particles (i.e. micron size) and to help in the calibration tasks of the instrument. To operate in the modified Twyman-Green interferometric configuration the second beam is attenuated and retarded and then recombined with the first one to be close to the $T_{o b}+e^{i \delta_{2}} T_{2}=0$ interference condition in the detector (some retardation should be introduced in the second beam to compensate mainly the non-ideal polarizing properties of the beam splitter).

\subsection{Microfluidic system}

The microfluidic channel consists of a fused silica capillary tubing (Polymicro Technologies) with an internal diameter of $100 \mu \mathrm{m}$. (Different diameters from 2 to $700 \mu \mathrm{m}$ can be also used). The protective polyimide coating from the silica tubing was thermally removed in the illuminated zone.

The nanoparticles solutions were prepared by dispersing wellcharacterized particles in ethanol. Particle aggregation was avoided by solution sonication. Solution was infused in the microfluidic channel using a syringe pump (NE100, New Era System) which was connected to a six port Rheodyne valve enabling to select the nanoparticles path. During the experiments the nanoparticles flow was kept constant.

\subsection{Data acquisition system}

The signal provided by the PMT detector is preamplified and transmitted to a PC waveform digitizer board (12 bit A/D, Spectrum MI3130). A LabVIEW program manages the A/D board, performs the Fourier analysis of the digitized signal and manages an $\mathrm{I} / \mathrm{O}$ module, which controls the amplitude modulation of the photoelastic modulator and the orientation of the motorized goniometers of the PSG and the analyzer.

\subsection{Calibration}

The first step of the alignment procedure is performed without the expanding lens and the pinhole and substituting the microfluidic channel by a mirror. The laser is aligned so that the beam passes perpendicularly through the centre of the collimating lens as well as the other optical elements (polarizers, beam splitter...). The mirrors are then set at approximately the same distance from the beam splitter and adjusted to obtain interference fringes in the region of overlap. Finally the expanding lens and the pinhole are replaced to obtain a collimated beam (see Ref. [8] for more details).

The orientation of the polarizer, photoelastic modulator and analyzer must be achieved with a sample providing good $R_{\omega}$ and $R_{2 \omega}$ ratios (i.e. by placing a quarter wave plate in front of the attenuator in the second beam). This procedure along with the procedures to determine the amplitude modulation of the modulator to work with the $\mathrm{J}_{0}(\mathrm{~A})=0$ condition and to determine the response coefficients have been described elsewhere [6].

The last step of the calibration deals with the precise positioning of the microfluidic channel in the focus of the objective. At this point the use of the CCD camera helps to focus the objective and to find the image plane in which the PMT detector has to be placed. 


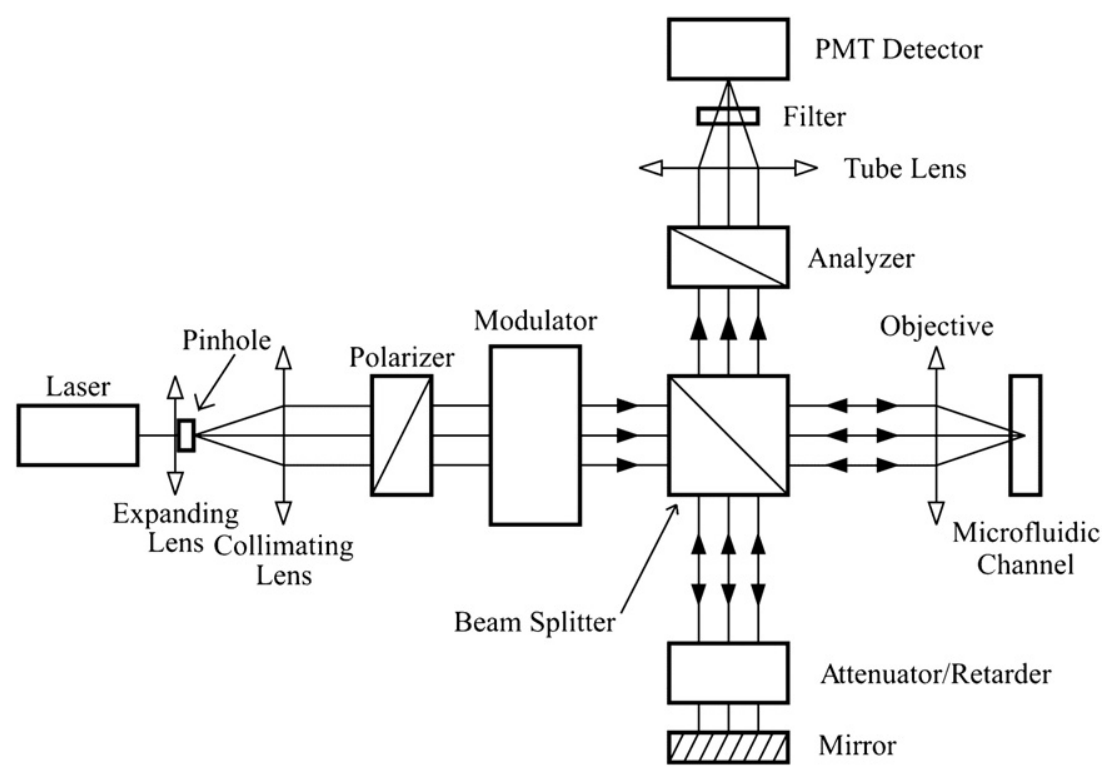

Fig. 1. Optical setup of the Nanopolar interferometer.

\section{Results and discussion}

\subsection{Microparticles detection}

$\mathrm{TiO}_{2}$ spherical particles of $2 \mu \mathrm{m}$ of diameter diluted in ethanol have produced a significant backscattered signal when crossing the focus of the microscope objective. Fig. 2 shows that the variation of the measured values in single mode operation is around $25 \%$ for the $\mathrm{dc}$ component ( $m_{\mathrm{I}(1,1)}-m_{\mathrm{I}(3,1)}$ value) and $50 \%$ for the $\mathrm{R}_{\omega}$ and $\mathrm{R}_{2 \omega}$ ratios. The results in the interferometric mode operation as a function of time
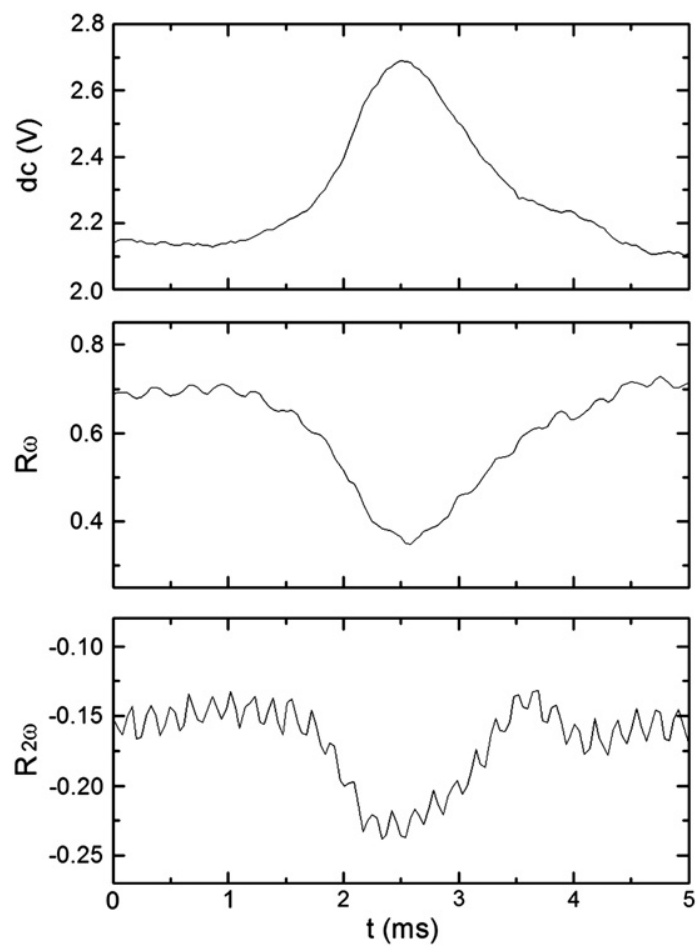

Fig. 2. Measured dc, $R_{\omega}$ and $R_{2 \omega}$ values as a function of time in the single mode for a $2 \mu \mathrm{m}$ particle of $\mathrm{TiO}_{2}$ passing through the laser focus. are displayed in Fig. 3. In this case the attenuator and retarder in the second beam were set to minimize the dc component; however a low frequency noise originating from mechanical vibrations and from the low visibility of the interference fringes hid the passage of the microparticles (see the undulation during the first milliseconds in the figure). The fact that the variations recorded for the dc component and the $R_{\omega}$ and $R_{2 \omega}$ ratios are similar to those observed in single mode operation, could indicate that the ideal interference condition was not attained in the measurements performed up to date. Further work is in progress to improve the overall interference of the light coming from the nanoparticles and the objective with the second beam.

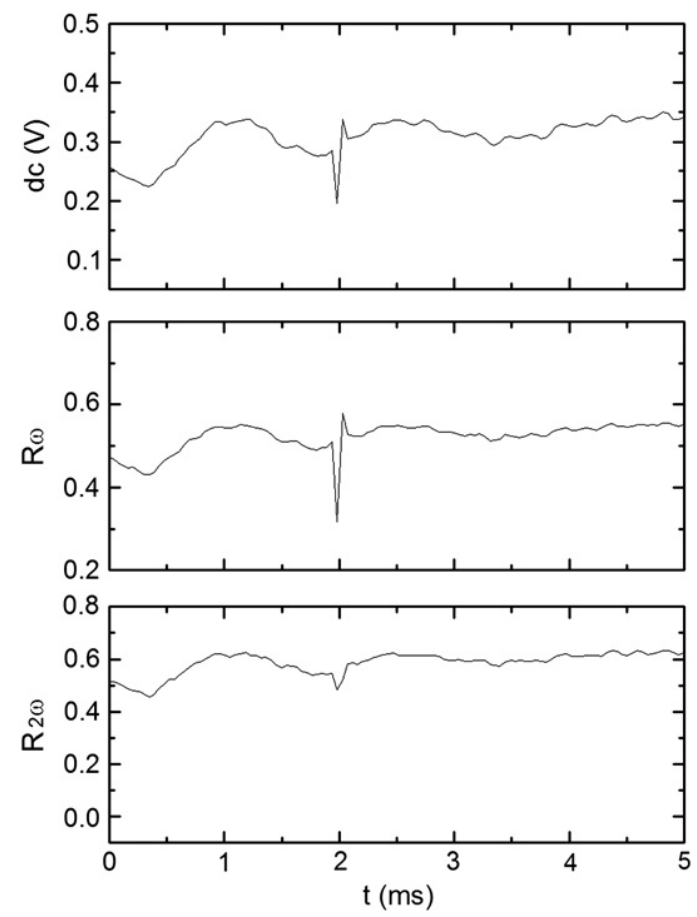

Fig. 3. Measured dc, $R_{\omega}$ and $R_{2 \omega}$ values as a function of time in the interferometric mode for a $2 \mu \mathrm{m}$ particle of $\mathrm{TiO}_{2}$ passing through the laser focus. 

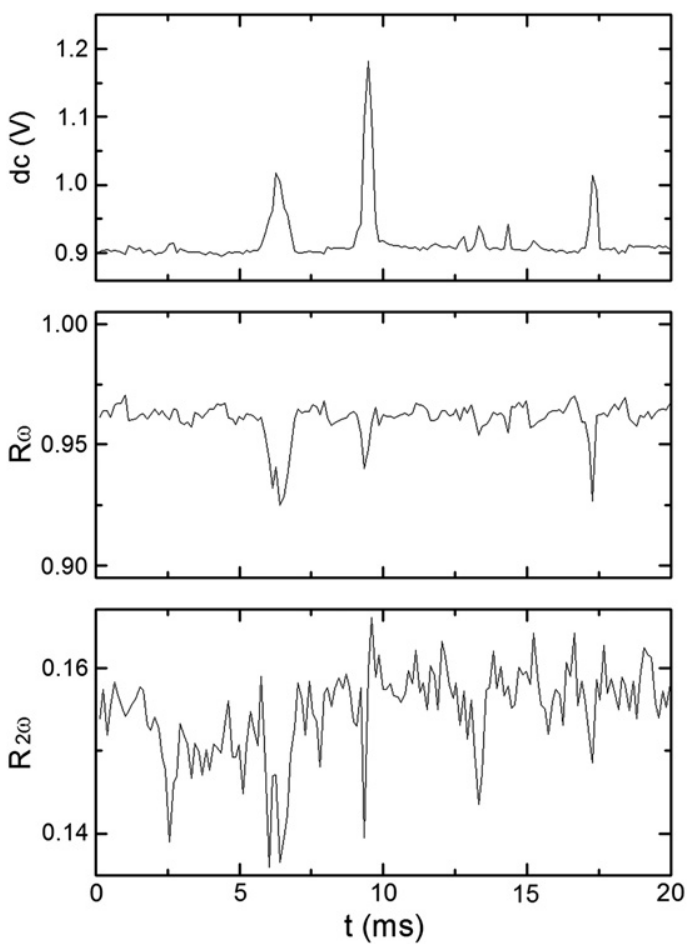

Fig. 4. Measured dc, $R_{\omega}$ and $R_{2 \omega}$ values as a function of time in the single mode for a $50 \mathrm{~nm}$ particle of Fe@C passing through the laser focus.

\subsection{Nanoparticles detection}

Fe-C core-shell (Fe@C) nanoparticles with sizes ranging from $10 \mathrm{~nm}$ to $50 \mathrm{~nm}$ have been used to test the detection limit of the

a

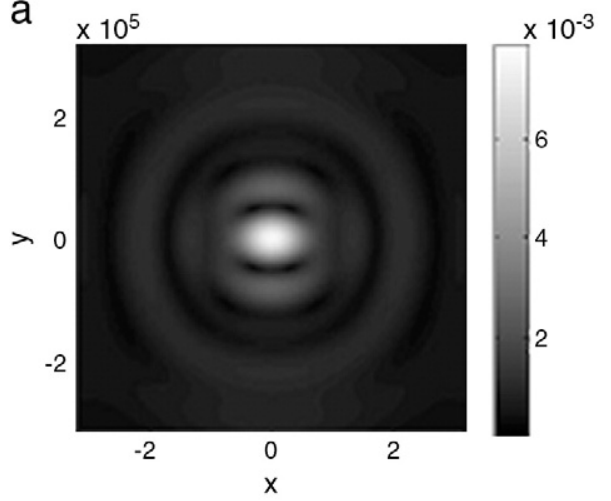

Nanopolar interferometer. In the single mode operation a significant variation of the $\mathrm{dc}$ and $\mathrm{R}_{\boldsymbol{\omega}}$ signals are obtained whereas the variation in the $R_{2 \omega}$ signal is hardly distinguishable from the noise level, as it can be inferred from Fig. 4 (the noise level corresponds to the signal detected when passing the carrying fluid without nanoparticles).

\subsection{Optical modelling}

The angular scattering symmetry for an arbitrary spherical particle leads to nonzero values for the matrix elements $m_{p(3,4)}$ and $m_{p(3,3)}$ and, in the case of a sphere sufficiently small that Rayleigh theory is applicable, $m_{p(3,4)}$ vanishes [5(b)]. For the measurements configuration used in the present work the ratios $R_{\omega}$ and $R_{2 \omega}$ are mainly related with $m_{p(3,4)}$ and $m_{p(3,3)}$ respectively. One interesting fact is that for Fe@C nanoparticles the measured variation in the ratio $R_{\omega}$ is significantly greater than in $\mathrm{R}_{2 \omega}$ suggesting a cross polarization effect of the polarization vectors when the beam is converted from divergent to parallel by the objective. Accordingly, this effect should be also present in the case of microparticles.

To support the previously mentioned consideration we have evaluated analytically the intensity and polarization of the backscattered light that is detected in our experiment. We have computed the electric fields for the light scattered in the back direction in the vicinity of the particle using the exact Mie theory [9]. Assuming the incident light propagating in the $z$ direction and linearly polarized along the $x$ axis, the program provides three 2D matrices for the $x, y$ and $z$ electric field components at points inside a $(x, y)$ plane placed at the back side of the particle and perpendicular to the incident direction. Fig. 5 shows the calculations for a $(x, y)$ square of $1.2 \mathrm{~mm}$ placed $320 \mu \mathrm{m}$ before a $\mathrm{TiO}_{2}$ microparticle of $2 \mu \mathrm{m}$ of diameter and a Fe nanoparticle of $50 \mathrm{~nm}$ of diameter (the position of the square plane corresponds to the objective front lens). The result of the computation shows that the maximum value for the $x$ component is about 5 times
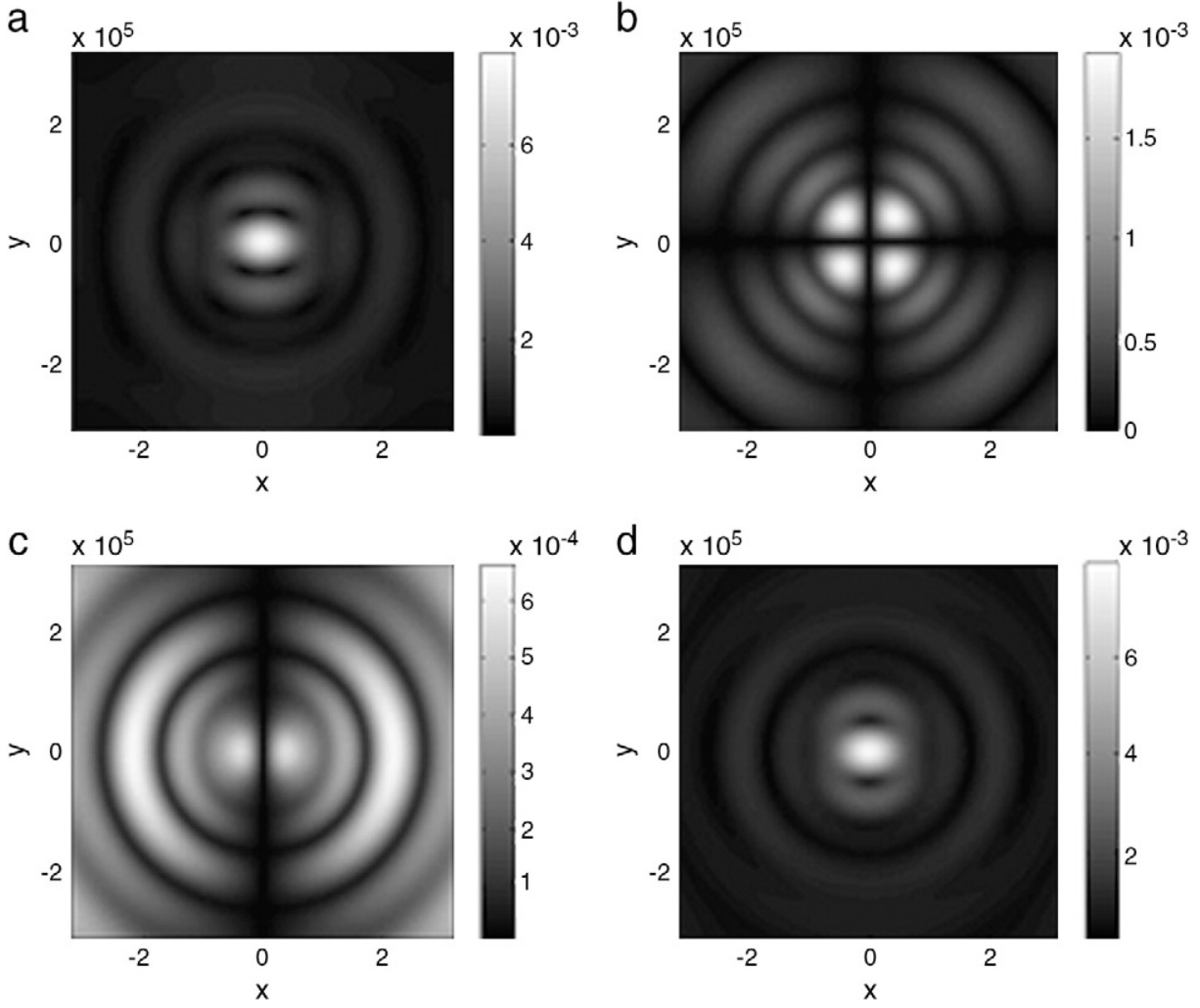

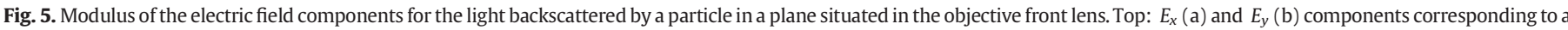

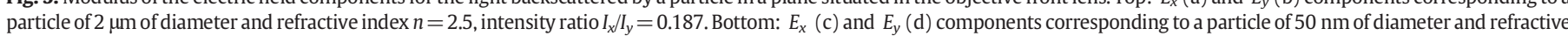
index $n=2.03-\mathrm{i} 2.86$, intensity ratio $I_{x} / I_{y}=0.079$. ( $x$ and $y$ distances are in $\mathrm{nm}$; the vertical bar indicates the relative electric field with respect to the incident electric field). 
greater than those for the $y$ component. The nonzero value for the $y$ component is an important result because it explains the observed cross polarization effect which is at the source of the significant variation of the $\mathrm{R}_{\omega}$ and the $\mathrm{R}_{2 \omega}$ ratios. The dependence of the $x$ and the $y$ electric field components on size, shape and refractive index of the particle will then make possible the characterization of individual particles with the technique tested in this work based on the Nanopolar interferometer. Furthermore, the improvement of the system by adding a second modulator $[10,11]$ will allow the measurement of the complete Mueller matrix $\mathrm{M}_{\mathrm{I}}$ and consequently a more detailed characterization of individual nanoscale particles.

\section{Conclusion}

The potential use of interferometric phase modulated ellipsometry to monitor in real time and to characterize single particles on the nanoscale level has been shown. The Nanopolar interferometric design, based on the collection of the backscattered light by the particles in the solid angle subtended by a microscope objective, yields remarkable $R_{\omega}$ and $R_{2 \omega}$ ratios arising from a cross polarization effect of the polarization vectors. The obtained results show that, at the present status of development, the sensitivity of the two modes is similar. However the possibility to combine the constructive interference of the electric fields scattered by the nanoparticles and the objective along with the destructive interference originated with the second beam opens a promising way to improve the sensitivity of the interferometric mode.

\section{Acknowledgments}

The authors acknowledge financial support from the Spanish government (CTQ2009-4674-02-01) and from AGAUR of the Generalitat de Catalunya (project 2009SGR00185, FEMAN Group). O. A. also acknowledges financial support from the Ministerio de Educación y Ciencia (MEC) of Spain (FPU AP2006-00193).

\section{References}

[1] K. Lindfords, T. Kalkbrenner, P. Stoller, V. Sandoghar, Phys. Rev. Lett. 93 (2004) 037401.

[2] F.V. Ignatovich, L. Novotny, Phys. Rev. Lett. 96 (2006) 013901

[3] J. Humlicek, in: H.G. Tompkins, E.A. Irine (Eds.), Handbook of Ellipsometry, William Andrew Publishing and Springer-Verlag GmbH \& Co. KG, 2005,, Chap. 1.

[4] M. Mansuripur, Classical Optics and its Applications, Cambridge University Press, 2002, Chap. 3.

[5] C. Bhoren, D. Huffman, Absorption and Scattering of Light by Small Particles, John Wiley \& Sons, New York, 1983, (a) Chap 3, p. 61. (b) Chap 13, p.414

[6] O. Acher, E. Bigan, E. Drévillon, Rev. Sci. Instrum. 60 (1989) 65.

[7] R.A. Chipman, 2nd ed., Handbook of Optics, vol. 2, McGraw-Hill Professional, 1994, Chap. 22.

[8] P. Hariharan, Academic Press, Boston, 1991, p. 175.

[9] K. Sendur, W. Challener, O. Mryasov, Opt. Express 16 (2008) 2874

[10] G.E. Jellison Jr., F.A. Modine, Appl. Opt. 36 (1997) 8190.

[11] O. Arteaga, A. Canillas, R. Purrello, J. Ribó, Opt. Lett. 34 (2009) 2177. 